\title{
Paradigm shift in hydrocephalus research in legacy of Dandy's pioneering work: rationale for third ventriculostomy in communicating hydrocephalus
}

\author{
Dan Greitz
}

Received: 23 January 2007 / Published online: 17 March 2007

(C) Springer-Verlag 2007

\begin{abstract}
Objective This study aims to question the generally accepted cerebrospinal fluid (CSF) bulk flow theory suggesting that the CSF is exclusively absorbed by the arachnoid villi and that the cause of hydrocephalus is a CSF absorption deficit. In addition, this study aims to briefly describe the new hydrodynamic concept of hydrocephalus and the rationale for endoscopic third ventriculostomy (ETV) in communicating hydrocephalus.

Critique The bulk flow theory has proven incapable of explaining the pivotal mechanisms behind communicating hydrocephalus. Thus, the theory is unable to explain why the ventricles enlarge, why the CSF pressure remains normal and why some patients improve after ETV.

Hydrodynamic concept of hydrocephalus Communicating hydrocephalus is caused by decreased intracranial compliance increasing the systolic pressure transmission into the brain parenchyma. The increased systolic pressure in the brain distends the brain towards the skull and simultaneously compresses the periventricular region of the brain against the ventricles. The final result is the predominant enlargement of the ventricles and narrowing of the subarachnoid space. The ETV reduces the increased systolic pressure in the brain simply by venting ventricular CSF through the stoma. The patent aqueduct in communicating hydrocephalus is too narrow to vent the CSF sufficiently.
\end{abstract}

D. Greitz $(\bowtie)$

Department of Neuroradiology, MR Research Center, Karolinska University Hospital, Bldg N8,

171 76, Stockholm, Sweden

e-mail: dan.greitz@karolinska.se
Keywords Cerebrospinal fluid · Compliance · ETV . Hydrocephalus · Normal-pressure hydrocephalus . Pathophysiology $\cdot$ Pulse pressure

\section{Introduction}

The modern era of hydrocephalus research began with the experimental studies of Dandy and Blackfan [3] in 1914, which, until today constitutes an unsurpassed contribution to this field. By plugging the aqueduct, they produced hydrocephalus in dogs. The division of hydrocephalus into the obstructive and communicating type originates from their work. The bulk flow theory and the concept of cerebrospinal fluid (CSF) malabsorption as the cause of hydrocephalus are also based on their experiment [3]. However, Dandy strongly opposed the view that idiopathic communicating hydrocephalus is caused by an obstruction at the arachnoid villi. Such an obstruction cannot cause a higher pressure in the ventricles than in the subarachnoid space but would rather dilate the subarachnoid space. He also objected to the view that the CSF is absorbed by the arachnoid villi and instead proved that the CSF is absorbed by the capillaries $[3,4]$. If this is true, the modern era of CSF and hydrocephalus research started and ended with Dandy, who left the pathophysiology of idiopathic communicating hydrocephalus unexplained for future exploration.

By going back to Dandy and following his lead of a capillary CSF absorption, a new understanding of hydrocephalus may be found. O'Connel [19] and Bering [1] suggested that increased pulse pressure in the ventricles is the cause of communicating hydrocephalus. In 1978, Di Rocco and Pettorossi [5] verified that an intraventricular pulsating balloon causes communicating hydrocephalus in sheep. In 1993, Greitz re-valuated the physiology of the 
CSF circulation and hydrocephalus by using flow sensitive magnetic resonance imaging (MRI) and radionuclide cisternography [9]. He suggested that the capillaries absorb the CSF and that the distending force in the production of chronic hydrocephalus is an increased systolic pulse pressure in brain tissue [14].

\section{The hydrodynamic concept of hydrocephalus}

Classification of hydrocephalus The classification is based on capillary absorption of the CSF [9-14]. Hydrocephalus is divided into two main groups, acute hydrocephalus and chronic hydrocephalus [14]. Acute hydrocephalus is caused by an intraventricular CSF obstruction in accordance with the conventional view. As opposed to that view, it is suggested that chronic hydrocephalus is caused by decreased intracranial compliance.

Causes of decreased intracranial compliance Although the etiology of the decreased intracranial compliance such as CSF obstructions at the foramen magnum and adhesions in the subarachnoid space after intracranial bleedings, trauma, operations and infections is well known, the etiology remains unknown in many idiopathic and congenital cases of hydrocephalus. However, the final stage of chronic hydrocephalus, independent of its etiology, is uniform. The final stage is characterised by narrow capacitance vessels and reduced cerebral blood volume. This reduces cerebral blood flow, reduces intracranial compliance and enhances the intracerebral pulse pressure.

Intracerebral pulse pressure theory of chronic hydrocephalus Chronic hydrocephalus consists of two subtypes, communicating hydrocephalus and chronic obstructive hydrocephalus [14]. The associated malabsorption of CSF is not involved as a causative factor in chronic hydrocephalus. The theory is based on one basic mechanism, i.e. that chronic hydrocephalus is caused by decreased intracranial compliance increasing the systolic pressure transmission into the brain parenchyma [9,10, 12-14]. The increased systolic pressure in the brain is the cause of the distension of the brain and ventricles. The systolic expansion of the brain distends the brain towards the skull and simultaneously compresses the periventricular region of the brain. The rationale result is the predominant enlargement of the ventricles and a narrowing of the subarachnoid space.

Rationale for ETV in communicating hydrocephalus As described above, communicating hydrocephalus is caused by decreased compliance increasing the systolic pressure transmission into the brain. The systolic force compresses the brain including the intracranial capacitance vessels, i.e. the cerebral veins and capillaries. This results in a vicious cycle with narrow capacitance vessels and significantly reduced cerebral blood volume that causes further decrease in intracranial compliance and further increase in intracranial pulse pressure. It is obvious that an endoscopic third ventriculostomy (ETV) may interrupt the vicious cycle and reduce the systolic pressure in the brain simply by venting ventricular CSF through the stoma. The patent aqueduct in communicating hydrocephalus is too narrow to vent the ventricular CSF sufficiently. By reducing the pulse pressure, the ETV also reduces the compression of the capacitance vessels thereby restoring some degree of venous compliance, which in turn reduces the intracerebral pulse pressure further.

ETV versus shunting The proposed concept opens a new avenue in that ETV may be an effective treatment also in communicating hydrocephalus [8, 16-18]. It thus constitutes an interchangeable alternative to shunting. The primary aim of the treatment of chronic hydrocephalus is to restore intracranial compliance, which is achieved by both treatments [14]. Effective shunting is based on a slight over-drainage of CSF that ultimately causes a direct and forced dilation of the compressed veins [14]. Therefore, shunting is more effective in increasing intracranial compliance than ETV where the dilating effect on the veins works indirectly by reducing the systolic compressing force on the veins. Because ETV provides a more physiological treatment and has less late complications than shunting, it may be of benefit to increase the number of patients finally treated by ETV $[2,6-8,15,17,18,20]$. In patients shunted under 1 year of age, ETV should be considered as an alternative treatment when they grow older. However, the final answer as to which is the optimal treatment must be based on randomized clinical studies.

\section{Discussion}

The aim of this paper is to briefly describe the hydrodynamic concept of hydrocephalus, which is based on Dandy's observation of capillary absorption of the CSF. Oi and Di Rocco [20] recently suggested that a minor CSF pathway through the brain parenchyma is the dominant CSF absorption site in the embryo, foetus and infant. The theory described here suggests that the minor pathway in the developing immature brain as well as the major pathway in adults are through the brain capillaries. It is beyond the scope of this brief communication to present the numerous physiological (and self-evident) evidences that the brain capillaries have the capacity to absorb fluids such as the CSF and its constituencies [3, 4, 9, 11, 12, 14]. This 
contrasts to the total lack of physiological evidence that the arachnoid villi can absorb the CSF. The CSF dynamics has been described in earlier published papers $[9,11,12,14]$.

The proposed concept indicates a paradigm shift in our view on hydrocephalus. It is suggested that chronic hydrocephalus is caused by decreased intracranial compliance rather than a CSF absorption deficit [14]. The associated CSF malabsorption is a secondary phenomenon to the decreased intracranial compliance. The only residue of the conventional view is acute hydrocephalus, which is caused by a CSF absorption deficit (of the periventricular capillaries). Normalpressure hydrocephalus, aqueductal stenosis as well as communicating hydrocephalus in infants and adults are typical manifestations within the spectrum of chronic hydrocephalus. It should be emphasised that chronic hydrocephalus is an unstable hemodynamic disorder occurring at normal or slightly increased mean intracranial pressure with superimposed vascular pressure waves that can be fatal.

The theory may thus have identified decreased intracranial compliance as a new and potential universal cause of chronic hydrocephalus. Decreased compliance is common to all different types of chronic hydrocephalus whatever their etiology. If the theory is correct, there is hope for improved treatment of chronic hydrocephalus of unknown etiology. How come then that treatment failure rather is the rule than the exception of this devastating condition? The reason may be that shunting and ETV essentially represents symptomatic treatments of hydrocephalus. To improve therapy, we have to find treatments that are more effective in increasing compliance in cases with unknown etiology or are directed against the cause of the decreased compliance in cases with known etiology.

\section{References}

1. Bering EA Jr (1962) Circulation of the cerebrospinal fluid. Demonstration of the choroid plexuses as the generator of the force for flow of fluid and ventricular enlargement. J Neurosurg $19: 405-413$

2. Cinalli G (1999) Alternatives to shunting. Childs Nerv Syst $15: 718-731$

3. Dandy WE, Blackfan KD (1914) Internal hydrocephalus. An experimental, clinical and pathological study. Am J Dis Child 8:406-482
4. Dandy WE (1929) Where is the CSF absorbed? JAMA 92:20122014

5. Di Rocco C, Pettorossi VE, Caldarelli MR, Mancinelli R, Velardi F (1978) Communicating hydrocephalus induced by mechanically increased amplitude of the intraventricular cerebrospinal fluid pressure: experimental studies. Exp Neurol 59:40-52

6. Di Rocco CL, Massimi L, Tamburrini G (2006) Shunts vs endoscopic third ventriculostomy in infants: are there different types and/or rates of complications? Childs Nerv Syst 22:15731589, DOI 10.1007/s00381-006-0194-4

7. Fukuhara T, Luciano MG (2001) Clinical features of late-onset idiopathic aqueductal stenosis. Surg Neurol 55:132-136

8. Gangemi M, Maiuri F, Buonamassa S, Collela G, de Divitiis E (2004) Endoscopic third ventriculostomy in idiopathic normal pressure hydrocephalus. Neurosurgery 55:129-134

9. Greitz D (1993) Cerebrospinal fluid circulation and associated intracranial dynamics. A radiologic investigation using MR imaging and radionuclide cisternography. Acta Radiol Suppl 386:1-23

10. Greitz D, Hannerz J, Rähn T, Bolander H, Ericsson A (1994) MR imaging of cerebrospinal fluid dynamics in health and disease. On the vascular pathogenesis of communicating hydrocephalus and benign intracranial hypertension. Acta Radiol 35:204-211

11. Greitz D, Hannerz J (1996) A proposed model of cerebrospinal fluid circulation: observations with radionuclide cisternography. Am J Neuroradiol 17:431-438

12. Greitz D, Greitz T, Hindmarsh TV (1997) A new view on the CSF-circulation with the potential for pharmacological treatment of childhood hydrocephalus. Acta Paediatr 86:125-132

13. Greitz D, Greitz T (1997) The pathogenesis and hemodynamics of hydrocephalus. A proposal for a new understanding. Int $\mathrm{J}$ Neuroradiol 3:367-375

14. Greitz D (2004) Radiological assessment of hydrocephalus: new theories and implications for therapy. Neurosurg Rev 27:145-165, (Review) DOI 10.1007/s10143-004-0326-9

15. Hellwig D, Grotenhuis JA, Tirakotai W, Riegel T, Schulte DM, Bauer BL, Bertalanffy H (2005) Endoscopic third ventriculostomy for obstructive hydrocephalus. Neurosurg Rev 28:1-34, DOI 10.1007/s10143-004-0365-2

16. Longatti PL, Fiorindi A, Martinuzzi A (2004) Failure of endoscopic third ventriculostomy in the treatment of idiopathic normal pressure hydrocephalus. Minim Invasive Neurosurg 47:342-345

17. Meier U (2003) Shunt operation versus endoscopic ventriculostomy in normal pressure hydrocephalus: diagnostics and outcome. Zentralbl Neurochir 64:19-23, (German)

18. Mitchell P, Mathew B (1999) Third ventriculostomy in normal pressure hydrocephalus. Br J Neurosurg 13:382-385

19. O'Connel JEA (1943) The vascular factor in intracranial pressure and the maintenance of the CSF circulation. Brain 66:204-228

20. Oi S, Di Rocco C (2006) Proposal of "evolution theory in cerebrospinal fluid dynamics" and minor pathway hydrocephalus in developing immature brain. Childs Nerv Syst 22:662-669, DOI 10.1007/s00381-005-0020-4 